\title{
Helium anion formation inside helium droplets ${ }^{\star}$
}

\author{
Elias Jabbour Al Maalouf, Julia Reitshammer, Anita Ribar, Paul Scheier, and Stephan Denifla \\ Institut für Ionenphysik und Angewandte Physik, Universität Innsbruck, Technikerstr. 25, 6020 Innsbruck, Austria
}

Received 26 February 2016 / Received in final form 20 May 2016

Published online 5 July 2016

(C) The Author(s) 2016. This article is published with open access at Springerlink.com

\begin{abstract}
The formation of $\mathrm{He}^{*-}$ is examined with improved electron energy resolution of about $100 \mathrm{meV}$ utilizing a hemispherical electron monochromator. The work presented provides a precise determination of the three previously determined resonance peak positions that significantly contribute to the formation of $\mathrm{He}^{*-}$ inside helium nanodroplets in the energy range from $20 \mathrm{eV}$ to $29.5 \mathrm{eV}$. In addition, a new feature is identified located at $27.69 \pm 0.18 \mathrm{eV}$ that we assign to the presence of $\mathrm{O}_{2}$ as a dopant inside the droplet. With increasing droplet size a small blue shift of the resonance positions is observed. Also for the relatively low electron currents used in the present study (i.e., $15-70 \mathrm{nA}$ ) a quadratic dependence of the $\mathrm{He}^{*-}$ ion yield on the electron current is observed.
\end{abstract}

\section{Introduction}

Helium droplets offer an interesting and exclusive environment for the study of physical and chemical phenomena [1-7]. This is due to the low temperature of helium droplets $(0.37 \mathrm{~K})$, their superfluidity and their capacity of efficient doping with a wide variety of atoms and molecules. Helium droplets provides an ideal cold matrix for spectroscopy that is interacting very little with the dopant [8]. Furthermore, the enormous cooling power of superfluid He has been reported to quench many fragmentation reactions and thus provides a method to investigate short-lived reaction intermediates [9]. For decades, the scientific community has been fascinated by the physics behind electron scattering from helium [10-14]. It has captured the attention of both the experimental and the theoretical communities that have attempted to better understand these fundamental reactions for years. Helium anions are some of the simplest metastable negative ions. The electron affinity of helium was first investigated in 1936 [15]. In the same year, helium anions $\mathrm{He}^{*-}$ were first detected by means of mass spectrometry [16]. Later, it was experimentally shown that $\mathrm{He}^{*-}$ can be formed by charge-exchange collisions [17]. $\mathrm{He}^{*-}$ has a lifetime of a few $100 \mu$ s which was determined experimentally utilizing storage rings $[18,19]$. Therefore the investigation of the physics and chemistry behind it prove to be challenging for this anion in the gas phase. Mauracher et al. [20] recently discovered that $\mathrm{He}^{*-}$ is efficiently formed upon electron

\footnotetext{
* Contribution to the Topical Issue "Advances in Positron and Electron Scattering", edited by Paulo Limao-Vieira, Gustavo Garcia, E. Krishnakumar, James Sullivan, Hajime Tanuma and Zoran Petrovic.

a e-mail: stephan.denifl@uibk.ac.at
}

irradiation of helium nanodroplets (HND) at specific resonance energies. In the meantime several novel processes have been ascribed to the presence of metastable $\mathrm{He}^{*-}$ in HND such as the formation and ejection of $\mathrm{He}^{+}$at electron energies below the ionization threshold of the free atom [21], or the self-assembly of salt nanocrystals from chemical reactions [22]. It has been shown that $\mathrm{He}^{*-}$ has a high mobility inside the helium droplet. This mobility enables it to find the dopant and transfer its negative charge and/or electronic excitation energy to it $[23,24]$. In a separate study, the dopant anions created at electron impact energies of about $22 \mathrm{eV}$ were attributed to the high mobility of $\mathrm{He}^{*-}$ inside the helium droplet [20]. It was shown that it is possible for $\mathrm{He}^{*-}$ to donate both loosely bound electrons to a fullerene cluster. Multiply charged cations formed inside helium droplets could be mostly attributed to $\mathrm{He}^{*-}$ acting as a reagent. Clear experimental evidence of the role of two bound states $\left(1 s 2 s 2 p^{4} \mathrm{P}\right)$ and the $\left(1 s 2 p^{2}\right.$ $\left.{ }^{4} \mathrm{P}\right)$ of $\mathrm{He}^{*-}$ was presented [25]. For undoped HND the intensity of the lowest energy resonance $(1 s 2 s 2 p)$ is exceeding all higher energy resonances by far which therefore only show up as shoulders in the high-energy tail of this dominant resonance. However, $\mathrm{He}^{*-}$ in the $(1 s 2 s 2 p)$ state is most strongly affected by the presence of an impurity, which was ascribed to the highest mobility of this electronic state of $\mathrm{He}^{*-}$. All previous experiments on $\mathrm{He}^{*-}$ from HND were performed with poor electron energy resolution which may affect the accurate assignment of both the position and width of the resonance structures.

In the present study we utilized a hemispherical electron monochromator to study the formation of the helium anion $\mathrm{He}^{*-}$ in helium droplets. The resonance peak positions as well as the width of the resonances for the formation of $\mathrm{He}^{*-}$ were determined with high energy resolution. In addition, we studied the dependence of these positions 
on the initial size of the neutral helium droplet, and the dependence on the ion yield with respect to the initial electron current.

\section{Experimental setup}

Pure helium nanodroplets were produced via the supersonic expansion of pressurized and pre-cooled He through a small orifice into ultra-high vacuum. A closed cycle cryostat was employed to cool down pure (99.9999\%) helium as it passes through a tube. The helium pressure inside the tube is regulated to 20 bar. At the end of the tube, the helium reaches a $5 \mu \mathrm{m}$ nozzle that is cooled down to low temperatures $(6.8-7.6 \mathrm{~K})$ using the same cryostat. When the supercritical fluid of helium expands through the nozzle to high vacuum, the liquid fragments into large droplets as a result of cavitation [26]. Evaporative cooling reduces the temperature of the helium droplets to $0.37 \mathrm{~K}[8]$. The resulting helium nanodroplets in this experiment had a mean size in the order of $10^{6}$ $10^{7}$ helium atoms [27]. We note that due to a small leak a small amount of air $\left(\sim 1 \times 10^{-5} \mathrm{~Pa}\right)$ was present in the droplet source chamber. The beam is then skimmed passing to a differentially pumped vacuum chamber via a $0.8 \mathrm{~mm}$ skimmer. More details on this helium droplet source can be found in [28]. In this differentially pumped chamber, the helium nanodroplet beam intersects with a high resolution electron beam [29]. At first the electrons are produced by a heated tungsten hairpin filament having a diameter of $0.125 \mathrm{~mm}$. After this a hemispherical electron monochromator (HEM) is employed to achieve an electron beam with high energy resolution. The electron beam resolution used for the present work was in the range between $90-110 \mathrm{meV}$. The resolution of the electron beam is determined by measuring the full width at half maximum (FWHM) of the anion efficiency curve of $\mathrm{SF}_{6}^{-}$upon electron attachment to gas phase $\mathrm{SF}_{6}$ having a maximum at $0 \mathrm{eV}$ [30]. The latter resonance was also used for the calibration of the energy scale. The electrons are measured at a Faraday cup using a pico-ammeter. In the present work, electron currents were in the range of 15-75 nA at the electron energy of $22 \mathrm{eV}$. Due to the interaction of the electron beam with the droplet beam, ions are formed. The resulting anions are guided towards a quadrupole mass filter where they are analyzed according to their mass to charge ratio. The detection is achieved by means of a Channeltron type secondary electron multiplier (KBL 510, Dr. Sjuts Optotechnik GmbH). A pulse counting system is used for analyzing and detecting the mass selected ionic products [31]. The channeltron is mounted $90^{\circ}$-off axis to the quadrupole. In the standard configuration the mass selected anions are bent by means of an electrostatic cylindrical sector (the outer electrode consists of a grid, while the inner electrode is a plate). This deflector allows the ions to hit the detector. In the present experiment with helium droplets, the problem occurs that for electron energies higher than $20 \mathrm{eV}$, neutral metastable species that are created are not affected by the quadrupole mass filter. These metastable species can collide with the

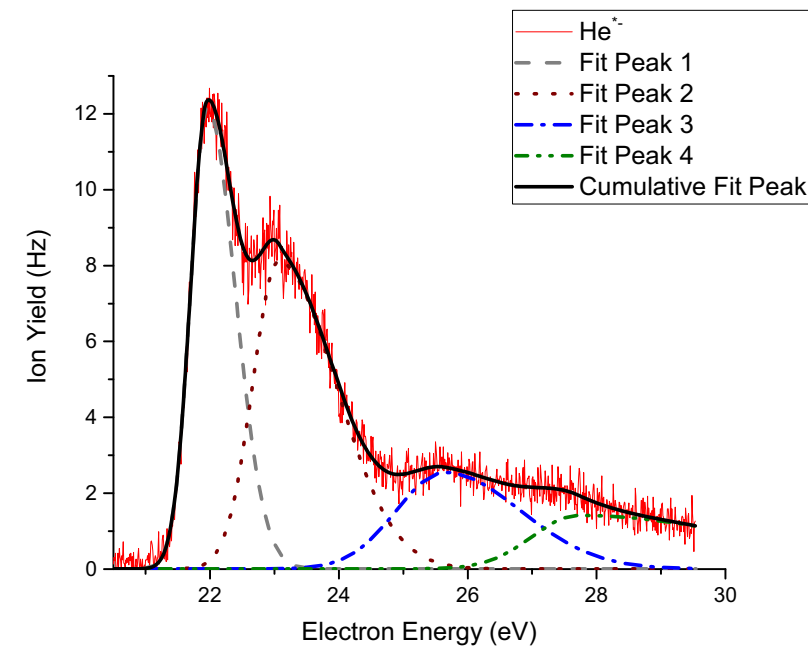

Fig. 1. Anion efficiency curve for $\mathrm{He}^{*-}$ upon electron collision of He droplets, weakly doped with air, and having an average size of $1.5 \times 10^{7}$ atoms (expansion conditions 20 bar, $6.8 \mathrm{~K}$ ). The smooth solid black line represents a fit via four bi-Gaussian curves (dashed lines) of the measured data.

grid of the electrostatic bender. As a result of such collisions, electrons and cations are formed and irrespective of the potential set on the detector one of these secondary species is accelerated to the detector. This signal adds to and often exceeds the ion yield of reactions that are investigated. To solve this problem, the cylindrical sector was replaced by a $90^{\circ}$ electrostatic quadrupole bender that allows the metastable particles to be dumped without contributing to the signal on the detector.

\section{Results and discussions}

The attachment of an electron to a helium atom of the nanodroplet proceeds at different resonance energies, each of which corresponds to a specific state. In the present work, clear experimental evidence of four different resonances for the formation of $\mathrm{He}^{*-}$ inside helium droplets is observed within a projectile electron energy range between $20 \mathrm{eV}$ and $29.5 \mathrm{eV}$. The anion yield of $\mathrm{He}^{*-}$ is plotted as a function of the projectile electron energy and presented in Figure 1.

Four distinct structures clearly appear in the anion efficiency curve of $\mathrm{He}^{*-}$. The threshold energy for the formation of $\mathrm{He}^{*-}$ corresponds to the onset of the first resonance found in the ion efficiency curve of $\mathrm{He}^{*-}$. The threshold was determined to be $21 \pm 0.2 \mathrm{eV}$ from the energy where ion yield is $0.1 \%$ of the maximum ion yield (vanishing current method). This threshold energy results from two components. The first is the energy required for the electron to penetrate the surface of the helium droplet. Very recently we reinvestigated the surface barrier energy with higher accuracy compared to previous studies [32]. This was possible by investigating low-energy electron attachment to the water dimer inside helium droplets with the HEM described in Section 2. We observed that the 
Table 1. Comparison between data measured with lower electron energy resolution $(\sim 1 \mathrm{eV}$ in $[20] ; \sim 0.25 \mathrm{eV}$ in [33]) and the present data with improved electron energy resolution $(\sim 110 \mathrm{meV})$.

\begin{tabular}{ccccc}
\hline Peak & $\begin{array}{c}\text { Position } \\
(\mathrm{eV})\end{array}$ & FWHM & $\begin{array}{c}\text { Position } \\
(\mathrm{eV})[20]\end{array}$ & $\begin{array}{c}\text { Position } \\
(\mathrm{eV})[33]\end{array}$ \\
\hline 1 & $21.98 \pm 0.05$ & 0.69 & $22.0 \pm 0.2$ & $22.33 \pm 0.1$ \\
2 & $22.96 \pm 0.07$ & 1.28 & $23.0 \pm 0.2$ & $23.34 \pm 0.1$ \\
3 & $25.78 \pm 0.10$ & 2.92 & $25.1 \pm 0.5$ & $25.93 \pm 0.1$ \\
4 & $27.69 \pm 0.18$ & 3.42 & - & - \\
\hline
\end{tabular}

value for the barrier gets higher when the droplet size increases. In the experiment for the present data the droplet size was relatively big so the value for this barrier is considered to be $0.98 \pm 0.05 \mathrm{eV}[32]$. The second is the energy needed to form the $\mathrm{He}^{*}(19.82 \mathrm{eV})$ from ground state helium [20]. Thus the total electron impact energy required to form $\mathrm{He}^{*-}$ inside a helium droplet adds up to $20.8 \mathrm{eV}$. This value is in agreement with the presently determined threshold value for $\mathrm{He}^{*-}$ at $21 \pm 0.2 \mathrm{eV}$. Electron attachment to pure helium droplets was previously investigated while utilizing an electron source having an energy resolution of about $250 \mathrm{meV}$. The onset of the anion efficiency curve for the formation of massive negatively charged HND was reported at $21.35 \mathrm{eV} \pm 0.25 \mathrm{eV}[33]$ for pure helium droplets with an average size $N=7 \times 10^{6}$. Another recent investigation on $\mathrm{He}^{*-}$ utilizing an electron source with a resolution $1 \mathrm{eV}$ reported a threshold at $21 \pm 0.5 \mathrm{eV}[20]$.

To further analyze the resonance features in the $\mathrm{He}^{*-}$ ion yield, we used quadruple bi-Gaussian fitting functions (see Fig. 1) and determined the position of the maxima as well as the FWHM for each of these four features (see Tab. 1). In contrast to the previous work [20] that employed a multiple Gaussian fit to investigate the peak positions, the bi-Gaussian fitting enables a better reproduction of the asymmetric shape of the four resonances. The four bi-Gaussian fits contributing to the reconstruction of the ion yield at $21.98 \pm 0.05 \mathrm{eV}, 22.96 \pm$ $0.07 \mathrm{eV}, 25.78 \pm 0.10 \mathrm{eV}, 27.69 \pm 0.18 \mathrm{eV}$. The observed peak positions confirm the previously identified peak positions $[20,33]$ with higher precision and accuracy along with the observation of a new structure positioned at $27.6 \mathrm{eV}$. Previously obtained peak positions are summarized with the ones from the present work in Table 1. The width (FWHM) of the four peaks increases gradually with the energy of the corresponding maxima, i.e., $0.69 \mathrm{eV}, 1.28 \mathrm{eV}$, $2.92 \mathrm{eV}$, and $3.42 \mathrm{eV}$. Peak 4 exhibits a long tail to the high-energy side. This broadening of the peaks can be attributed to the large number of closely lying states of $\mathrm{He}^{*-}$ that lead to the overlapping of more resonances as the projectile electron energy increases.

$\mathrm{He}^{*-}$ is heliophilic [25] and thus is expected to remain inside the HND after its formation. In order to eject this anion form the droplet we propose the presence of a second negatively charged species in the same droplet. Thus at least two electrons have to interact with the HND for the detection of $\mathrm{He}^{*-}$. The first electron is required to form
$\mathrm{He}^{*-}$ via inelastic scattering at one $\mathrm{He}$ atom. Thereby electronically excited helium, $\mathrm{He}^{*}$, is formed and a lowenergy electron. The latter interacts with the surrounding He via Pauli repulsion and forms a vacuum bubble. At the same time it polarizes the He atoms and to a much larger extent the metastable $\mathrm{He}^{*}$. The scattered electron and the $\mathrm{He}^{*}$ attract each other and finally leads to the attachment of the electron to the $\mathrm{He}^{*}$ forming $\mathrm{He}^{*-}\left(1 s 2 s 2 p{ }^{4} \mathrm{P}\right)$. The second electron may form another $\mathrm{He}^{*-}$. Alternatively it may remain as an electron bubble, particularly if the presence of a charged species suppresses the recombination of the electron bubble and the He* Coulomb repulsion pushes one $\mathrm{He}^{*-}$ out of the droplet. In case of an electron bubble and one $\mathrm{He}^{*-}$ the higher mobility of $\mathrm{He}^{*-}$ will favor the ejection of the latter. Therefore, the detection of $\mathrm{He}^{*-}$ from large $\mathrm{He}$ droplets requires a double electron interaction process. In such case, the ion yield is quadratically enhanced when the number of projectile electrons increases. It was shown in previous experiments employing electron beams with electron currents in the range of $\mu \mathrm{A}$ that the ion yield of $\mathrm{He}^{*-}$ formation at a fixed electron impact energy shows a quadratic dependence on the electron current $[20,25]$. In the present work, we confirm this quadratic dependence using an electron source producing electron currents in the $\mathrm{nA}$ range. A plot of the ion yield of the $\mathrm{He}^{*-}$ formation at the projectile electron energy of $22 \mathrm{eV}$ as a function of the electron current is presented in Figure 2. This plot confirms the proposed reaction mechanism that the ejection of $\mathrm{He}^{*-}$ upon electron irradiation of large helium droplets is solely possible via a two-electron scattering process.

The first peak appearing around $22 \mathrm{eV}$ is labeled as peak 1 in Figure 1. This peak is assigned to the formation of $\mathrm{He}^{*-}\left(1 s 2 s 2 p{ }^{4} \mathrm{P}\right)$ by the attachment of the scattered electron (bubble) to the exited $\mathrm{He}^{*}\left(1 s 2 s^{3} \mathrm{~S}\right)$. We note that in the case of doped helium droplets, ion induced dipole interaction between the $\mathrm{He}^{*-}$ and the dopant will attract the two species and lead to several possible reactions, that remove the $\mathrm{He}^{*-}$ and ionize the dopant [25]. Some of the possible reactions that can take place between $\mathrm{He}^{*-}$ and the dopant are:

$$
\begin{aligned}
\mathrm{He}^{*-}+\mathrm{AB} \longrightarrow \mathrm{He}^{*}+\mathrm{AB}^{-} & \\
\mathrm{He}^{*-}+\mathrm{AB} \longrightarrow \mathrm{He}^{+}+\mathrm{AB}^{2-*} & \\
\mathrm{He}^{*-}+\mathrm{AB} \longrightarrow \mathrm{He}+\mathrm{AB}^{*-} & \longrightarrow \mathrm{AB}^{+}+2 \mathrm{e} \\
& \longrightarrow \mathrm{A}^{+}+\mathrm{B}+2 \mathrm{e} \\
& \longrightarrow \mathrm{AB}^{2+}+3 \mathrm{e} \\
& \longrightarrow \mathrm{A}^{+}+\mathrm{B}^{+}+3 \mathrm{e}
\end{aligned}
$$

But only the first two reactions are leading to the formation of an anion, required to push a $\mathrm{He}^{*-}$ out of the droplet. The second resonant structure around $23 \mathrm{eV}$ is labelled as peak 2 in Figure 1. This resonance corresponds to the formation of $\mathrm{He}^{*-}$ via electron attachment to a higher excited state of the helium atom, i.e., $\mathrm{He}^{*}\left(1 s 2 p^{3} \mathrm{P}\right)$. The energy required to form this excited state from the ground state of neutral helium is $20.96 \mathrm{eV}$ [34]. The resulting helium anion upon electron attachment is $\mathrm{He}^{*-}\left(1 s 2 p^{2}{ }^{4} \mathrm{P}\right)$ 


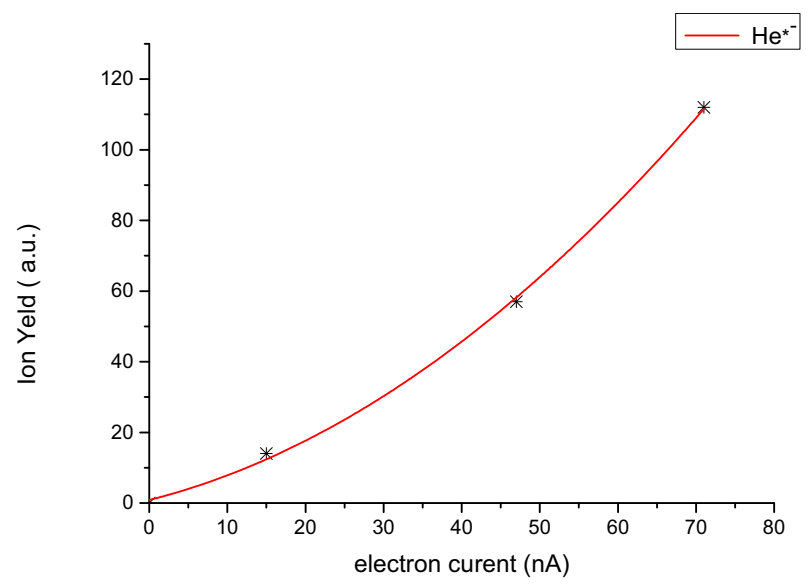

Fig. 2. The ion yield of $\mathrm{He}^{*-}$ as a function of the projectile electron current at a nozzle temperature of $6.8 \mathrm{~K}$ and an electron energy of $22 \mathrm{eV}$.

and has a life time of $0.1 \mu \mathrm{s}$ [34]. This life time is too short for detection in our apparatus with flight times between the formation and detection in the order of several $\mu \mathrm{s}$. Thus we conclude that this higher lying state of $\mathrm{He}^{*-}$ will convert into $\mathrm{He}^{*-}\left(1 s 2 s 2 p{ }^{4} \mathrm{P}\right)$ before ejection from the droplet. In the same range of energies for this resonance structure the first state of $\mathrm{He}_{2}^{*-}$ is also accessible [20]. Previous experiments showed that in presence of dopants $\left(\mathrm{SF}_{6}\right)$ inside the helium droplet, the intensity of the first two peaks decreases with respect to the other resonance structures at higher energies [25].

The structure appearing with a maximum at around $25.78 \mathrm{eV}$ is labeled as peak 3 in Figure 1. Two different mechanisms may contribute to the appearance of this structure. The first mechanism is electron attachment to the excited state $\mathrm{He}^{*}\left(1 s 3 \mathrm{~s}^{3} \mathrm{~S}\right)$ of an isolated helium atom. This state is at an energy of $22.72 \mathrm{eV}$ [35] above the ground state of a helium atom. The higher width of this peak also indicates contributions of higher excited states that are located between $22.72 \mathrm{eV}$ and the ionization continuum at $24.59 \mathrm{eV}$ but cannot be resolved due to the width of each individual contribution. Due to the fact that most of this structure is above the ionization threshold of helium in the gas phase $(24.58 \mathrm{eV})$, another mechanism could contribute to this third resonance [36]. This mechanism involves at first the formation of $\mathrm{He}^{+}\left({ }^{2} \mathrm{~S}\right)$ via electron ionization of He $\left({ }^{1} \mathrm{~S}\right)$. Then, the two secondary electrons (the ejected and the scattered one) get trapped in the surrounding He matrix via loss of energy upon bubble formation and a lack of energy to reach the conduction band of the helium droplet. Both electrons will be attracted by the cation and upon recombination with the $\mathrm{He}^{+}\left({ }^{2} \mathrm{~S}\right)$ a highly excited anion in a quartet state $\mathrm{He}^{*-}\left({ }^{4} \mathrm{P}\right)$ will be formed as illustrated in reaction (4).

$$
\mathrm{e}+\mathrm{He}\left({ }^{1} \mathrm{~S}\right) \longrightarrow 2 \mathrm{e}+\mathrm{He}^{+}\left({ }^{2} \mathrm{~S}\right) \longrightarrow \mathrm{He}^{*-}\left({ }^{4} \mathrm{P}\right) .
$$

The structure at highest electron energy that we identify has a peak at about $27.7 \mathrm{eV}$ and is labeled in Figure 1 as peak 4 . The contributing mechanism for the appearance of this peak may be assigned to the higher excited Rydberg

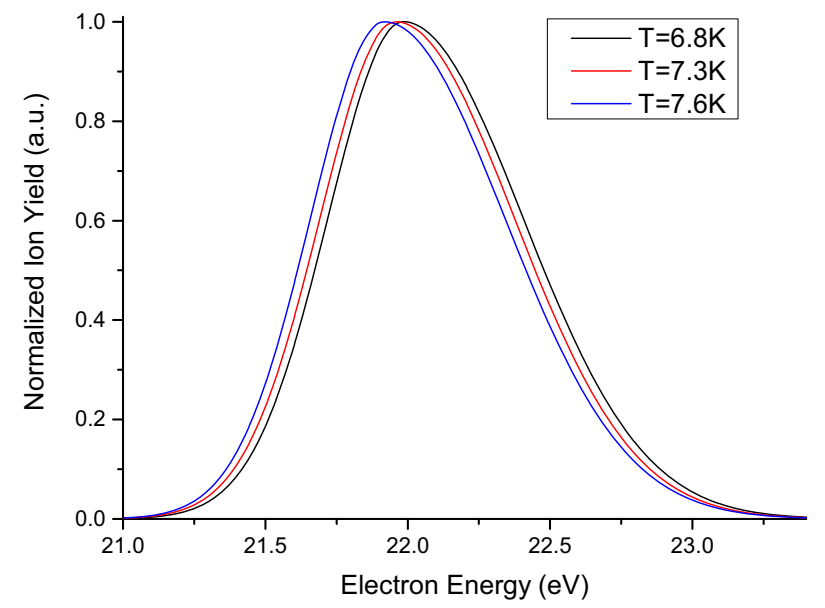

Fig. 3. Normalized bi-Gaussian curve obtained from fitting the anion efficiency curve of $\mathrm{He}^{*-}$ at $22 \mathrm{eV}$ at different nozzle temperatures. A blue-shift of $70 \mathrm{meV}$ is observed when changing the temperature of the He before the expansion from $7.6 \mathrm{~K}$ to $6.8 \mathrm{~K}$.

Table 2. The $\mathrm{He}^{*-}$ resonance peak position at different nozzle temperatures.

\begin{tabular}{ccc}
\hline Nozzle temperature (K) & Peak 1 position (eV) & FWHM \\
\hline 6.8 & $21.98 \pm 0.05$ & $0.69 \pm 0.05$ \\
7.3 & $21.95 \pm 0.05$ & $0.69 \pm 0.05$ \\
7.6 & $21.91 \pm 0.05$ & $0.69 \pm 0.05$ \\
\hline
\end{tabular}

states of the helium atom. The energy range is in the continuum of the ionization of helium. A contribution could come from the mechanism shown in reaction (4). Another possible mechanism is the formation of the $\mathrm{He}^{*-}$ accompanied by the formation of another anion. From the energy range of peak 4 we propose that the other anion is $\mathrm{O}^{-}$ formed via dissociative attachment of an electron (DEA) that has been inelastically scattered at one of the He atoms to an $\mathrm{O}_{2}$ dopant. $\mathrm{O}^{-}$formation from $\mathrm{O}_{2}$ exhibits in gas phase a resonance at $6.5 \pm 0.5 \mathrm{eV}$ [37]. Thus the projectile electron requires a energy of $19.82 \mathrm{eV}\left(\mathrm{He}^{*}\right)+1.24 \mathrm{eV}$ (energy barrier for electrons to enter the droplet) $+6.5 \mathrm{eV}$ $\left(\right.$ DEA to $\left.\mathrm{O}_{2}\right)=27.56 \mathrm{eV}$.

Anion efficiency curves for $\mathrm{He}^{*-}$ were recorded covering the energy range of peak 1 , while setting the nozzle temperature to three different values $(6.8 \mathrm{~K}, 7.3 \mathrm{~K}$, $7.6 \mathrm{~K})$. The pressure of the helium gas was kept constant at 20 bar. The HEM was kept at the same conditions with the electron current around $70 \mathrm{nA}$. Using bi-Gaussian functions, the anion efficiency curves were fitted and the position and width of peak 1, were determined for the three different temperatures (see Fig. 3).

As the temperature of the nozzle drops, the mean droplet size of the formed helium droplets increases to larger values. A reduction of the temperature of the nozzle from $7.6 \mathrm{~K}$ to $6.8 \mathrm{~K}$ results in an increase of the average droplet size from $N=6 \times 10^{6}$ to $2 \times 10^{7}$ helium atoms [27]. For higher average droplet size the position of peak 1 is slightly shifted by about $70 \mathrm{meV}$ to higher energy. Such a blue-shift may be ascribed to the change 
in the surface barrier of the droplets with different droplet sizes as predicted by Rosenblit and Jortner [38]. Also for large negatively charged He droplets Henne and Toennies reported an energy shift of almost $0.5 \mathrm{eV}$ of the peak positions of the resonances when changing the average droplet size from $9.3 \times 10^{3}$ to $1.54 \times 10^{7}$. The same group also reported a blue-shift of the energy barrier for an electron to penetrate a helium droplet with increasing average droplet size [39]. Therefore, we tentatively assign the blue-shift of the resonance position for the formation of $\mathrm{He}^{*-}$ with increasing average droplet size also to the rise of the energy barrier for the electron entering the He droplet.

\section{Conclusion}

In the experiments conducted in the present study, the positions of the $\mathrm{He}^{*-}$ resonance peaks were determined with high precision and accuracy. The data were fitted using four bi-Gaussian curves allowing for the reproduction of the asymmetry of the resonance structures and thus providing a more accurate determination of the maximum peak positions. Evidence of the formation of $\mathrm{He}^{*-}$ via an intermediate $\mathrm{He}^{+}$that captures both low-energy secondary electrons was observed. The quadratic dependence of the yield of $\mathrm{He}^{*-}$ on the electron current confirms the proposed model of the ejection of the swift $\mathrm{He}^{*-}$ from a doubly or multiply charged anionic He droplet. The second negatively charged species can either be an electron bubble, another $\mathrm{He}^{*}$ or a dopant anion. In the present study the latter is formed via dissociative electron attachment to $\mathrm{O}_{2}$ which leads to a unique resonance at $27.7 \mathrm{eV}$.

In highest appreciation, this work is dedicated to Prof. Michael Allan, for that we learned from him about electron scattering and electron attachment. This work was supported by FWF, Vienna, Austria (P24443).

\section{References}

1. A. Slenczka, B. Dick, M. Hartmann, J.P. Toennies, J. Chem. Phys. 115, 10199 (2001)

2. M. Rosenblit, J. Jortner, J. Chem. Phys. 124, 194505 (2006)

3. F. Zappa, S. Denifl, I. Mähr, A. Bacher, O. Echt, T.D. Märk, P. Scheier, J. Am. Chem. Soc. 130, 5573 (2008)

4. S. Denifl, F. Zappa, A. Mauracher, F. Ferreira da Silva, A. Bacher, O. Echt, T.D. Märk, D.K. Bohme, P. Scheier, ChemPhysChem 9, 1387 (2008)

5. S. Denifl et al., Angew. Chem. Int. Ed. 48, 8940 (2009)

6. L.F. Gomez et al., Science 345, 906 (2014)

7. K. Nauta, R.E. Miller, Science 283, 1895 (1999)

8. J.P. Toennies, A.F. Vilesov, Angew. Chem. Int. Ed. 43, $2622(2004)$

9. W.K. Lewis, B.E. Applegate, J. Sztáray, B. Sztáray, T. Baer, R.J. Bemish, R.E. Miller, J. Am. Chem. Soc. 126, $11283(2004)$

10. G.J. Schulz, Phys. Rev. Lett. 10, 104 (1963)
11. M. Allan, J. Phys. B 25, 1559 (1992)

12. K.R. Asmis, M. Allan, J. Phys. B 30, L167 (1997)

13. K.R. Asmis, M. Allan, J. Phys. B 30, 1961 (1997)

14. M. Allan, J. Phys. B 33, L215 (2000)

15. T. Wu, Philos. Mag. 22, 837 (1936)

16. J.W. Hiby, Ann. Phys. 426, 473 (1939)

17. P.M. Windham, P.J. Joseph, J.A. Weinman, Phys. Rev. 109, 1193 (1958)

18. U.V. Pedersen, M. Hyde, S.P. Müller, T. Andersen, Phys. Rev. A 64, 012503 (2001)

19. H.T. Schmidt et al., J. Phys.: Conf. Ser. 388, 012006 (2012)

20. A. Mauracher, M. Daxner, J. Postler, S.E. Huber, S. Denifl, P. Scheier, J.P. Toennies, J. Phys. Chem. Lett. 5, 2444 (2014)

21. M. Renzler, M. Daxner, N. Weinberger, S. Denifl, P. Scheier, O. Echt, Phys. Chem. Chem. Phys. 16, 22466 (2014)

22. M. Daxner, S. Denifl, P. Scheier, A.M. Ellis, Angew. Chem. Int. Ed. 53, 13528 (2014)

23. P. Reinhed et al., Phys. Rev. Lett. 103, 213002 (2009)

24. A. Mauracher, M. Daxner, S.E. Huber, J. Postler, M. Renzler, S. Denifl, P. Scheier, A.M. Ellis, Angew. Chem. Int. Ed. 53, 13794 (2014)

25. A. Mauracher, M. Daxner, S.E. Huber, J. Postler, M. Renzler, S. Denifl, P. Scheier, A.M. Ellis, J. Chem. Phys. 142, 104306 (2015)

26. E.L. Knuth, U. Henne, J. Chem. Phys. 110, 2664 (1999)

27. L.F. Gomez, E. Loginov, R. Sliter, A.F. Vilesov, J. Chem. Phys. 135, 154201 (2011)

28. L. An der Lan, P. Bartl, C. Leidlmair, H. Schöbel, R. Jochum, S. Denifl, T.D. Märk, A.M. Ellis, P. Scheier, J. Chem. Phys. 135, 044309 (2011)

29. S. Denifl, S. Ptasińska, B. Sonnweber, P. Scheier, D. Liu, F. Hagelberg, J. Mack, L.T. Scott, T.D. Märk, J. Chem. Phys. 123, 104308 (2005)

30. M. Braun, S. Marienfeld, M.W. Ruf, H. Hotop, J. Phys. B 42, 125202 (2009)

31. G. Hanel, T. Fiegele, A. Stamatovic, T. Märk, Int. J. Mass Spectrom. 205, 65 (2001)

32. E. Jabbour Al Maalouf, P. Scheier, E. Illenberger, S. Denifl, in preparation (2016)

33. U. Henne, J.P. Toennies, J. Chem. Phys. 108, 9327 (1998)

34. A.A. Radzig, B.M. Smirnov, Reference Data on Atoms, Molecules, and Ions (Springer-Verlag, Berlin, Heidelberg, 1985)

35. W. Demtröder, Atoms, Molecules and Photons (Springer, Berlin, Heidelberg, 2010)

36. S.E. Huber, A. Mauracher, Molecul. Phys. 112, 794 (2014)

37. D. Nandi, V.S. Prabhudesai, E. Krishnakumar, A. Chatterjee, Rev. Sci. Instrum. 76, 053107 (2005)

38. M. Rosenblit, J. Jortner, J. Chem. Phys. 124, 194506 (2006)

39. K. Martini, J. Toennies, C. Winkler, Chem. Phys. Lett. 178, 429 (1991)

Open Access This is an open access article distributed under the terms of the Creative Commons Attribution License (http://creativecommons.org/licenses/by/4.0), which permits unrestricted use, distribution, and reproduction in any medium, provided the original work is properly cited. 